\title{
Natural antibiotic susceptibility of Providencia stuartii, $P$. rettgeri, $P$. alcalifaciens and $P$. rustigianii strains
}

\author{
INGO STOCK and B. WIEDEMANN \\ Pharmazeutische Mikrobiologie, University of Bonn, Germany
}

\begin{abstract}
The natural antibiotic susceptibility of 38 Providencia rettgeri, $35 P$. stuartii, $23 P$. alcalifaciens and $20 P$. rustigianii strains was examined. MIC values were determined by a microdilution procedure and evaluated by a table calculation programme. $P$. stuartii was the least susceptible Providencia sp. and was naturally resistant to tetracyclines, some penicillins, older cephalosporins, sulphamethoxazole and fosfomycin and to antibiotics to which other species of Enterobacteriaceae are also resistant. It was naturally sensitive to modern penicillins and cephalosporins, carbapenems and aztreonam, but its susceptibility to aminoglycosides and quinolones was difficult to assess. $P$. alcalifaciens and $\boldsymbol{P}$. rustigianii strains were the most susceptible Providencia spp. They were naturally sensitive or intermediate to tetracyclines and sensitive to aminoglycosides and quinolones. Susceptibility to sparfloxacin, biapenem and sulphamethoxazole permitted the discrimination of $P$. alcalifaciens and $P$. rustigianii strains. The natural antibiotic susceptibility of $P$. rettgeri strains was between that of $P$. stuartii and that of the other providenciae. $P$. rettgeri was resistant to tetracyclines and fosfomycin, but more susceptible to aminoglycosides, quinolones, fosfomycin and numerous $\beta$-lactam antibiotics than $P$ stuartii. A database is described of the natural antibiotic susceptibilities of Providencia spp. It can be used for the validation of antibiotic susceptibility test results of these micro-organisms.
\end{abstract}

\section{Introduction}

Providencia spp. are typical aetiological agents of nosocomial infections and are now of significant medical interest. This is attributed to the increase in numbers of elderly patients and improvements in medical care, resulting in a higher incidence of immunocompromised patients and increasing numbers of nosocomial infections.

From a medical point of view, $P$. stuartii is the most important species of the genus. P. stuartii has been identified frequently as a cause of nosocomial urinary tract infection (UTI). Predisposing circumstances like catheterisation and intubation favour the establishment of UTIs with this bacterium. Less frequently it also causes respiratory and skin infections and is isolated occasionally from surgical wounds. $P$. stuartii is a

Received 30 Sept. 1997; revised version accepted 21 Nov. 1997.

Corresponding author: Professor B. Wiedemann. genomically homogeneous species and is found predominantly in the urine [1] and in the groin areas of hospitalised patients, but also in water samples [2].

P. rettgeri is also an important infectious agent of the human urinary tract, but is isolated less frequently than $P$. stuartii. In part this is because of the susceptibility of $P$. rettgeri to the aminoglycosides often used in the treatment of UTIs (and to which $P$. stuartii is less susceptible). $P$. rettgeri has been isolated from poultry, the faeces of reptiles and amphibians and from surface waters and, therefore, seems to be widely distributed in nature. There is evidence that $P$. rettgeri is also a pathogen for a wide range of animals. Recently, meningitis in crocodiles due to $P$. rettgeri has been documented [3].

In contrast to these species, which generally do not contribute to gastrointestinal infections of man, certain strains of $P$. alcalifaciens are invasive enteric pathogens that can cause diarrhoea $[4,5]$ including travellers' diarrhoea [6]. The species consists of two genospecies [7]. In 1983, a fourth species, P. rustigianii was 
described for strains that had previously been called $P$. alcalifaciens biovar $3[8,9]$. P. rustigianii can be isolated from human faeces and can colonise the human intestinal tract, but its medical significance awaits further study. The Providencia sp. described most recently is $P$. heimbachae [10]. There is no evidence to date that this bacterium is of significance to man, because all isolates have been from penguin faeces and an aborted bovine fetus [11].

Many Providencia isolates, in particular $P$. stuartii strains, are resistant to numerous antibiotics. With the exception of $P$. stuartii, knowledge about the antibiotic susceptibility of Providencia spp. is relatively incomplete. This is partly because many laboratories identify providenciae only to the genus level and susceptibility testing is documented with only a few antibiotics. P. stuartii is the most resistant species of the genus and is generally considered to be an antibiotic multiresistant bacterium [1]. Like most other strains of the Proteeae, $P$. stuartii is resistant to polymyxin B, colistin and nitrofurantoin. The species is considered to be resistant also to some aminoglycosides and penicillins, older cephalosporins, tetracyclines and chloramphenicol. P. rettgeri and P. alcalifaciens strains are 'less resistant' than $P$. stuartii to aminoglycosides, cephalosporins and substituted penicillins $[1,11]$. Cornaglia and co-workers found no significant differences in antibiotic susceptibility between $P$. rettgeri and $P$. alcalifaciens strains [12]. Otherwise, multiresistant $P$. rettgeri strains have been described [13, 14] and are not rare. The increasing significance of infections with Providencia spp. necessitates a more detailed elucidation of differences in antibiotic susceptibility between Providencia spp.

The object of this study was to establish a database for the natural susceptibility to a wide range of antibiotics of all Providencia spp. pathogenic to man. The data from 116 Providencia strains tested with 71 antibiotics can be valuable for the validation of routine susceptibility test results.

\section{Materials and methods}

\section{Bacterial strains}

A total of 116 Providencia strains was examined. The strains were clinical isolates from the culture collection of H. Grimm (Weingarten, Germany) and B. Vocht (Moers, Germany) and from our culture collection. The latter originated from a multicentre study of the Paul Ehrlich Society conducted in 1986 with 30 centres in Germany, Switzerland and Austria and from the collection of MERLIN-Diagnostika (Bornheim, Germany). The majority of the strains submitted by $\mathrm{H}$. Grimm, B. Vocht and MERLIN were recent isolates and were collected between 1990 and 1997. Copy strains (more than the isolate/patient) and duplicate strains from the same outbreak were excluded.

\section{Identification}

All strains were identified to the species level with a commercial identification system for Enterobacteriaceae (Micronaut-E, MERLIN-Diagnostika, Bornheim, Germany) and a rapid identification system for gramnegative and gram-positive bacteria (Micronaut-IDS, MERLIN-Diagnostika). Both identification systems test key biochemical reactions of bacteria with clinical significance. The inoculum for the identification tests was a suspension from an overnight culture on solid medium in physiological saline solution at $10^{6} \mathrm{cfu} / \mathrm{ml}$ for the Micronaut $\mathrm{E}$ system and $10^{8} \mathrm{cfu} /$ $\mathrm{ml}$ for the Micronaut-IDS system. The incubation times were $24 \mathrm{~h}$ (Micronaut-E and agar plates) or $5 \mathrm{~h}$ (Micronaut-IDS) at $36^{\circ} \mathrm{C}\left( \pm 1^{\circ}\right)$. Fermentation of Dgalactose was tested on bromocresol purple agar (Difco) supplemented with galactose $6 \mathrm{~g} / \mathrm{L}$. All identification tests were repeated to confirm the results.

\section{Antibiotic susceptibility testing}

Antibiotic susceptibility was tested by a microdilution procedure in Isosensitest Broth (Oxoid). After inoculation of antibiotic containing microtitration plates (MERLIN-Diagnostika) with $100 \mu \mathrm{l}$ of appropriate bacterial suspension $\left(\sim 10^{5} \mathrm{cfu} / \mathrm{ml}\right)$ and incubation for $24 \mathrm{~h}$ at $36^{\circ} \mathrm{C}\left( \pm 1^{\circ}\right)$ MIC values were determined with a photometer (Labsystems Multiskan Multisoft). MIC data were evaluted with EXCEL (Microsoft).

\section{Evaluation of natural antibiotic susceptibility}

Plotting the MIC of a particular antibiotic for one species against the number of strains found with the respective MIC usually results in a bimodal distribution. One peak with relatively low MICs represents the natural population and one peak with higher MICs represents the strains with acquired (secondary) resistance. Analysis of the MIC distribution of all strains of one species for each antibiotic permitted determination of the biological thresholds, which limit the natural population at high MICs but not those strains with secondary (acquired) resistance (Fig. 1a and b). Whether the MIC values of the natural population were above or below the breakpoints of the standards, which assess the clinical susceptibility was investigated. Determination of whether the natural population was clinically sensitive, intermediate or resistant was by application of French (SFM), Swedish (SIR), German (DIN), American (NCCLS) and English standards (BSAC). When the natural population was sensitive or intermediate according to the cited standards, it was described as naturally sensitive (Fig. 1a) or naturally intermediate, respectively. When the natural population was resistant, it was described as naturally (primary or intrinsically) resistant (Fig. 1b). 
a
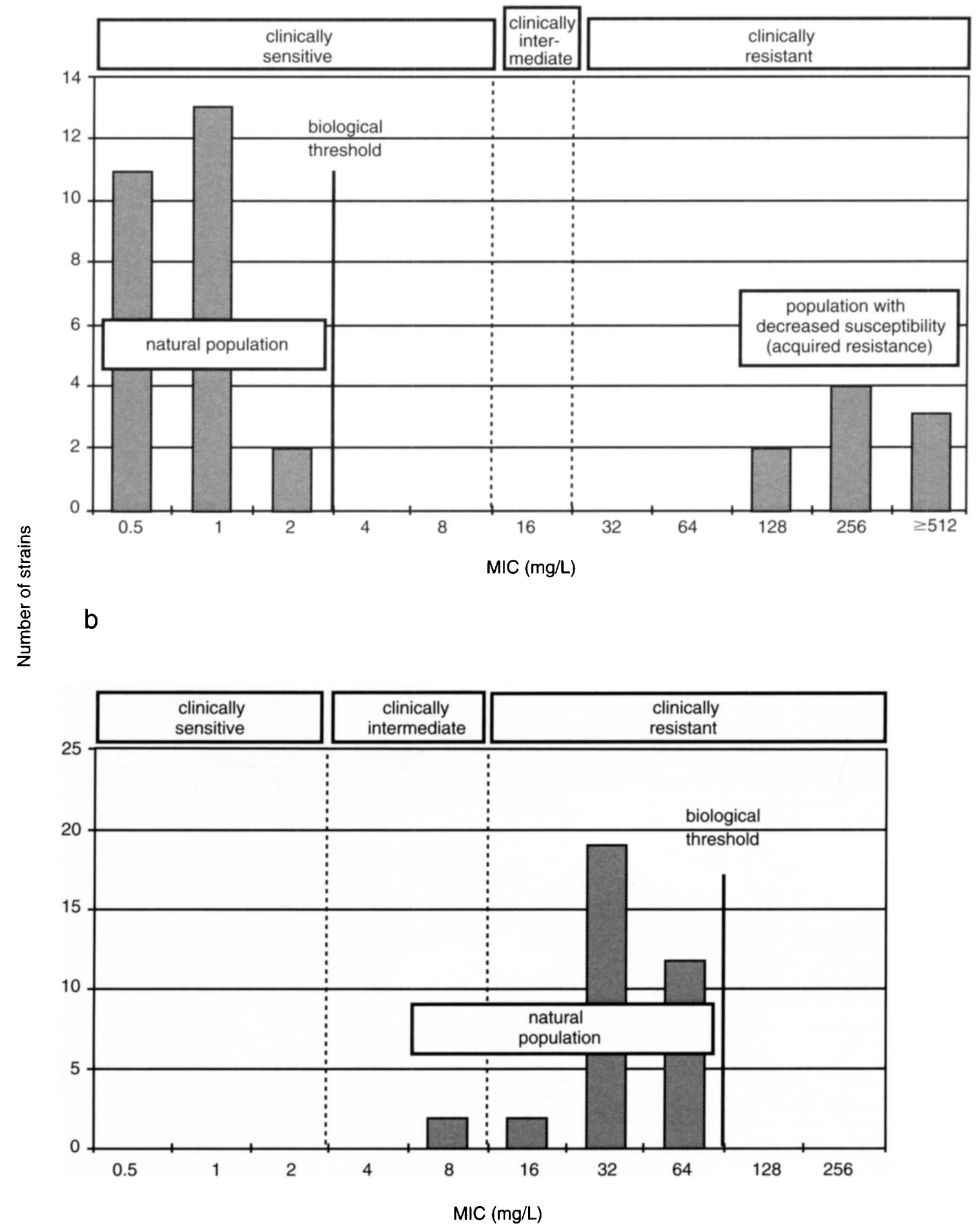

Fig. 1. (a) Susceptibility of $P$. stuartii to kanamycin. The biological threshold limits the natural population at high MICs excluding the strains with acquired resistance (population with decreased susceptibility). The biological threshold of $P$. stuartii for kanamycin was $2 \mathrm{mg} / \mathrm{L}$. The MIC values of the acquired resistant population were $128->256 \mathrm{mg} / \mathrm{L}$ (see Table 2). The clinical assessment of MIC data is shown according to French standards (SFM). Clinical thresholds are indicated by dotted lines. P. stuartii was naturally sensitive to kanamycin, as the natural population was clinically sensitive according to SFM. (b) Susceptibility of $P$. stuartii towards amoxycillin in combination with clavulanic acid (AMX/CLAV). The biological threshold of $P$. stuartii for AMX/CLAV was $64 \mathrm{mg} / \mathrm{L}$. Strains with acquired resistance were not found (see Table 2). The clinical assessment of MIC data is shown according to German standards (DIN). $P$. stuartii was naturally resistant to AMX/CLAV, as the natural population was clinically resistant according to DIN. 


\section{Results}

\section{Identification of strains}

The biochemical features of the Providencia strains examined are shown in Table 1. Thirty-five strains were identified as $P$. stuartii, 38 as $P$. rettgeri, 23 as $P$. alcalifaciens and 20 as $P$. rustigianii. Generally, there were no significant differences between the results and those reported by Farmer [15], although the results of mannose and trehalose fermentation were read in the Micronaut-IDS system after $5 \mathrm{~h}$ whereas Farmer read these results after incubation for 2 days. Aesculin hydrolysis, urease production, fermentation of rhamnose, adonitol, myo-inositol, trehalose, D-galactose and the nagase and phosphodiesterase tests (cleavage of $p$ nitrophenyl-acetylglucosamine and 2 -deoxythymidine$5 '$ - $p$-nitrophenylphosphate, respectively) were the most suitable tests to distinguish the Providencia spp. (Table 1).

\section{Natural antibiotic sensitivity and primary resistance}

The MIC distributions of the Providencia strains tested are presented in Table 2 . There were significant differences in antibiotic susceptibility between the different Providencia spp. Antibiotics with discriminating features for Providencia spp. included tetracyclines and fosfomycin. A summary of the natural antibiotic sensitivities and primary resistances of Providencia spp. is shown in Table 3. Strains with acquired resistance were found in all species (Table 2).

\section{Discussion}

P. stuartii has been called a multiresistant species [1]. It was found, that $P$. stuartii was naturally resistant to a wide range of antibiotics, e.g., tetracyclines, some penicillins and older cephalosporins, to which some

Table 1. Biochemical features of Providencia spp.

\begin{tabular}{|c|c|c|c|c|c|c|c|c|}
\hline \multirow[b]{3}{*}{ Biochemical test } & \multicolumn{8}{|c|}{ Positive reactions $(\%)$} \\
\hline & \multicolumn{2}{|c|}{ P. rettgeri } & \multicolumn{2}{|c|}{ P. stuartii } & \multicolumn{2}{|c|}{ P. alcalifaciens } & \multicolumn{2}{|c|}{ P. rustigianii } \\
\hline & I & II & I & II & I & II & I & II \\
\hline 1. Amino acid deaminase ${ }^{0}$ & 100 & 98 & 97 & 96 & 100 & 98 & 100 & 100 \\
\hline 2. Hydrogen sulphide production & 0 & 0 & 3 & 0 & 0 & 0 & 0 & 0 \\
\hline 3. $\beta$-Glucosidase ${ }^{1}$ & 68 & 35 & 0 & 0 & 0 & 0 & 25 & 0 \\
\hline 4. Tryptophanase ${ }^{2}$ & 92 & 99 & 97 & 98 & 87 & 99 & 95 & 98 \\
\hline 5. Urease & 95 & 98 & 40 & 30 & 0 & 0 & 0 & 0 \\
\hline 6. Lysine decarboxylase & 0 & 0 & 0 & 0 & 0 & 0 & 0 & 0 \\
\hline 7. Ornithine decarboxylase & 0 & 0 & 0 & 0 & 0 & 1 & 0 & 0 \\
\hline 8. Arginine dihydrolase & 0 & 0 & 0 & 0 & 0 & 0 & 0 & 0 \\
\hline 9. Glucose fermentation & 100 & 100 & 100 & 100 & 100 & 100 & 100 & 100 \\
\hline 10. Citrate assimilation ${ }^{3}$ & 100 & 95 & 100 & 93 & 100 & 98 & 100 & 15 \\
\hline 11. Malonate assimilation & 0 & 0 & 0 & 0 & 0 & 0 & 10 & 0 \\
\hline 12. Voges Proskauer reation & 0 & 0 & 0 & 0 & 0 & 0 & 0 & 0 \\
\hline 13. Rhamnose fermentation & 45 & 70 & $\mathbf{0}$ & 0 & 0 & 0 & 0 & 0 \\
\hline 14. Sucrose fermentation & 3 & 15 & 29 & 50 & 0 & 15 & 0 & 35 \\
\hline 15. Adonitol fermentation & 100 & 100 & $\mathbf{0}$ & 5 & 100 & 98 & 0 & 0 \\
\hline 16. (Myo)-inositol fermentation & 100 & 90 & 94 & 95 & $\mathbf{0}$ & 1 & 0 & 0 \\
\hline 17. Xylose fermentation & 8 & 10 & 6 & 7 & 13 & 1 & 25 & 0 \\
\hline 18. Sorbitol fermentation & 0 & 1 & 0 & 1 & 0 & 1 & 0 & 0 \\
\hline 19. $\beta$-Galactosidase ${ }^{4}$ & 3 & 5 & 0 & 10 & 0 & 1 & 0 & 0 \\
\hline 20. $\beta$-Xylosidase ${ }^{5}$ & 0 & ns & 0 & ns & 0 & ns & 0 & ns \\
\hline 21. $\beta$-Glucuronidase ${ }^{6}$ & 0 & ns & 0 & ns & 0 & ns & 0 & ns \\
\hline 22. Proline amidase & 0 & ns & 0 & ns & 0 & ns & 0 & ns \\
\hline 23. Pyrase & 0 & $\mathrm{~ns}$ & 0 & ns & 0 & ns & 0 & ns \\
\hline 24. Hydroxyproline amidase & 0 & ns & 0 & ns & 0 & ns & 0 & ns \\
\hline 25. Peptidase & 0 & $\mathrm{~ns}$ & 0 & ns & 0 & ns & 0 & ns \\
\hline 26. $\alpha$-Glucosidase & 3 & $\mathrm{~ns}$ & 0 & ns & 0 & ns & 0 & ns \\
\hline 27. Nagase (chitinase) ${ }^{7}$ & $\mathbf{0}$ & ns & 100 & ns & 17 & ns & 10 & ns \\
\hline 28. $\alpha$-Galactosidase & 0 & ns & 0 & ns & 0 & ns & 0 & ns \\
\hline 29. Phosphodiesterase $^{8}$ & 76 & ns & $\mathbf{0}$ & ns & 61 & ns & 15 & ns \\
\hline 30. L-Alanine aminopeptidase & 100 & ns & 100 & ns & 100 & ns & 100 & ns \\
\hline 31. Mannose fermentation & 100 & 100 & 100 & 100 & 100 & 100 & 100 & 100 \\
\hline 32. Trehalose fermentation & $\mathbf{0}$ & 0 & 97 & 98 & 0 & 2 & 0 & 0 \\
\hline 33. D-Galactose fermentation & 100 & ns & 100 & ns & 0 & ns & 100 & ns \\
\hline
\end{tabular}

The results (I) are contrasted with the data of Farmer (II) [15]. Most of the reactions were either included in the panels of the Micronaut-E (1-21.) or the Micronaut-IDS identification system of MERLIN (22-32).

Key discriminating reactions are given in bold print. ${ }^{0}$ tryptophan deaminase (MERLIN), phenylalanine deaminase [Farmer]; ${ }^{1}$ hydrolysis of aesculin; ' 2 indole production; ${ }^{3}$ mixture of Simmons' and Christensen citrate (MERLIN), Simmons' citrate [Farmer]; cleavage of ${ }^{4}$ orthonitrophenyl- $\beta$-galactopyranoside (ONPG), ${ }^{5}$ ortho-nitrophenyl- $\beta$-xyloside (ONPX), ${ }^{6}$ para-nitrophenyl- $\beta$-glucuronide (PGUR), ${ }^{7}$ para-nitrophenylacetylglucosamine, ${ }^{8} 2^{\prime}$-deoxythymidine-5' -para-nitrophenylphosphate; ns, not stated. 


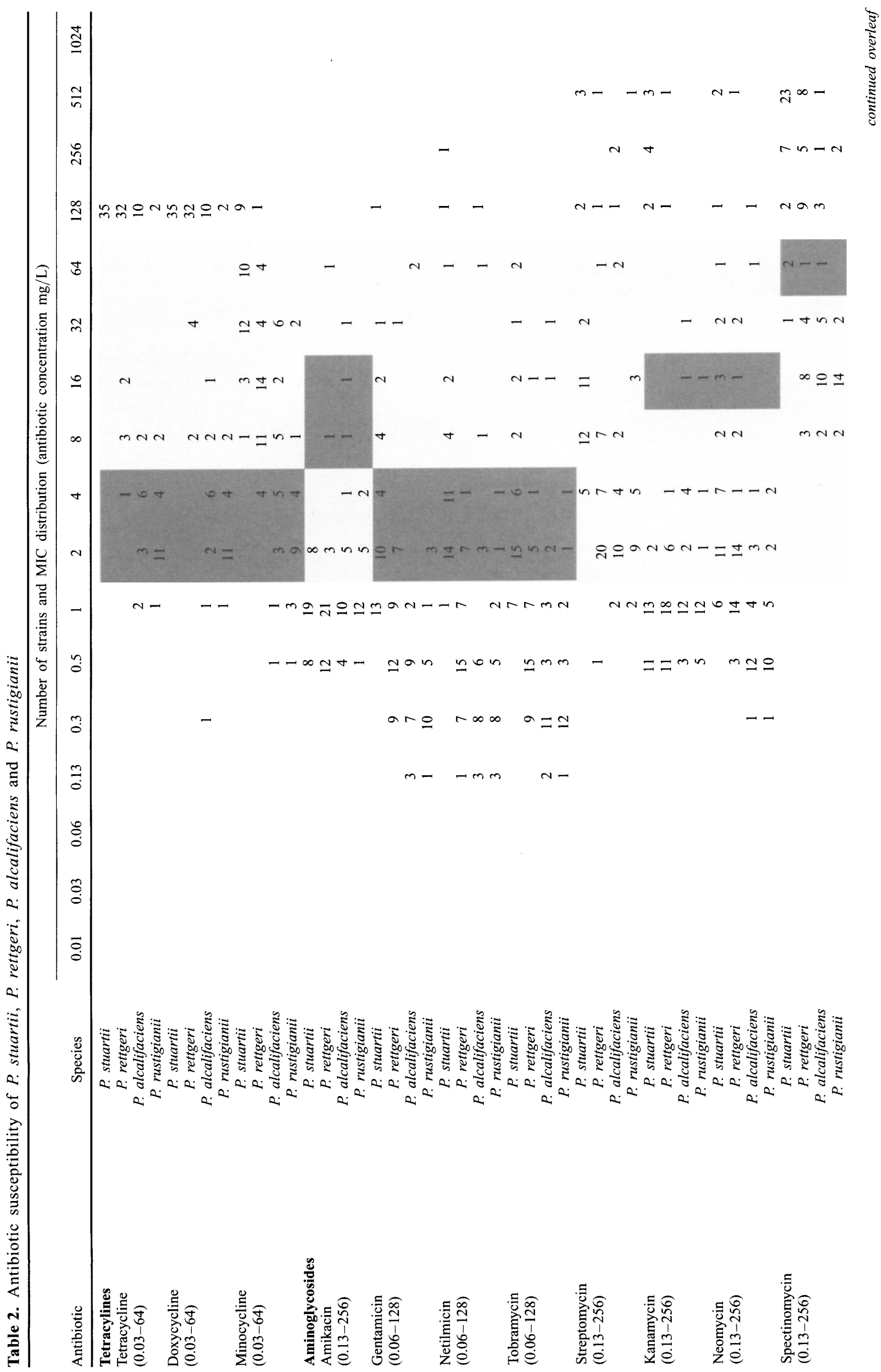




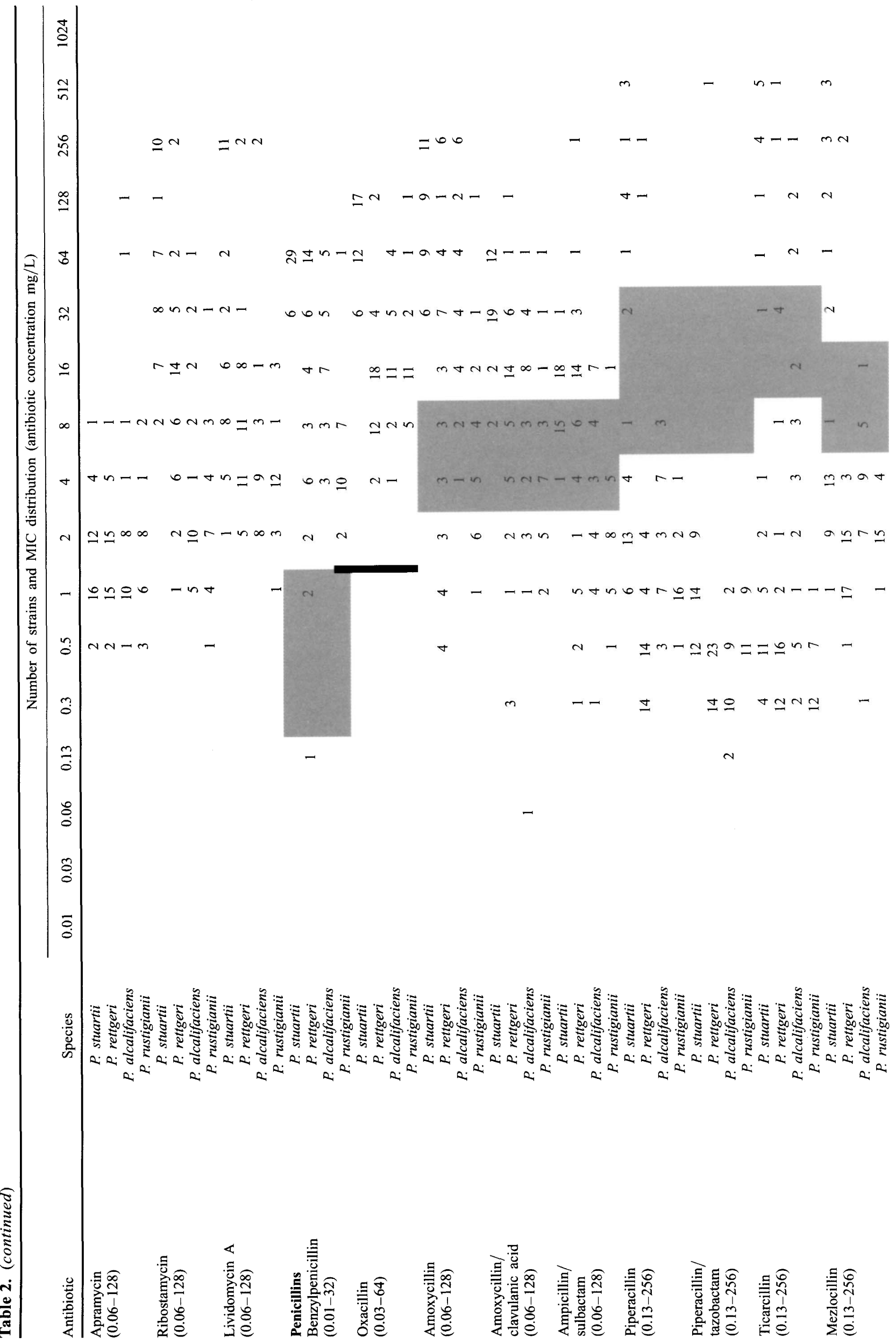




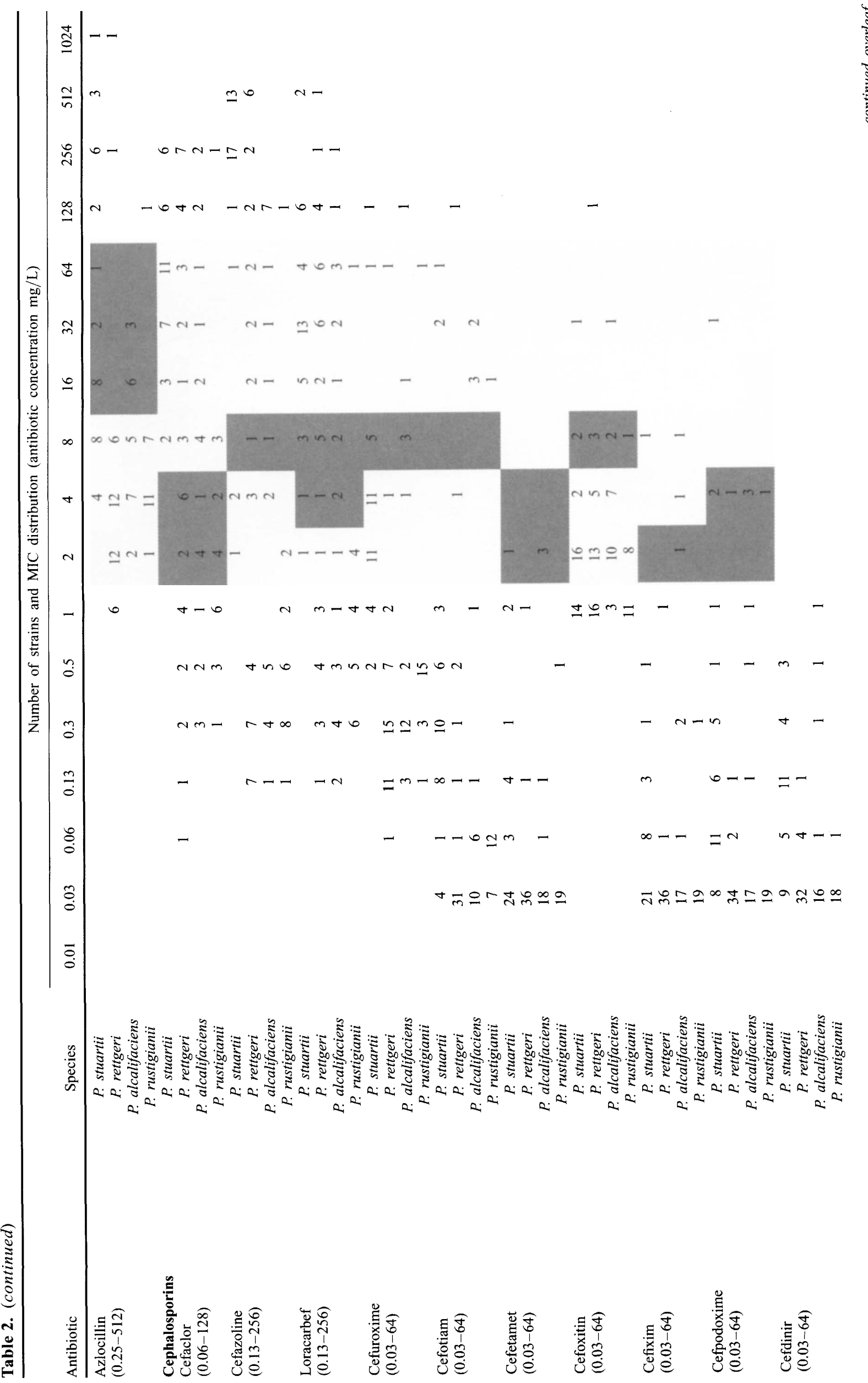




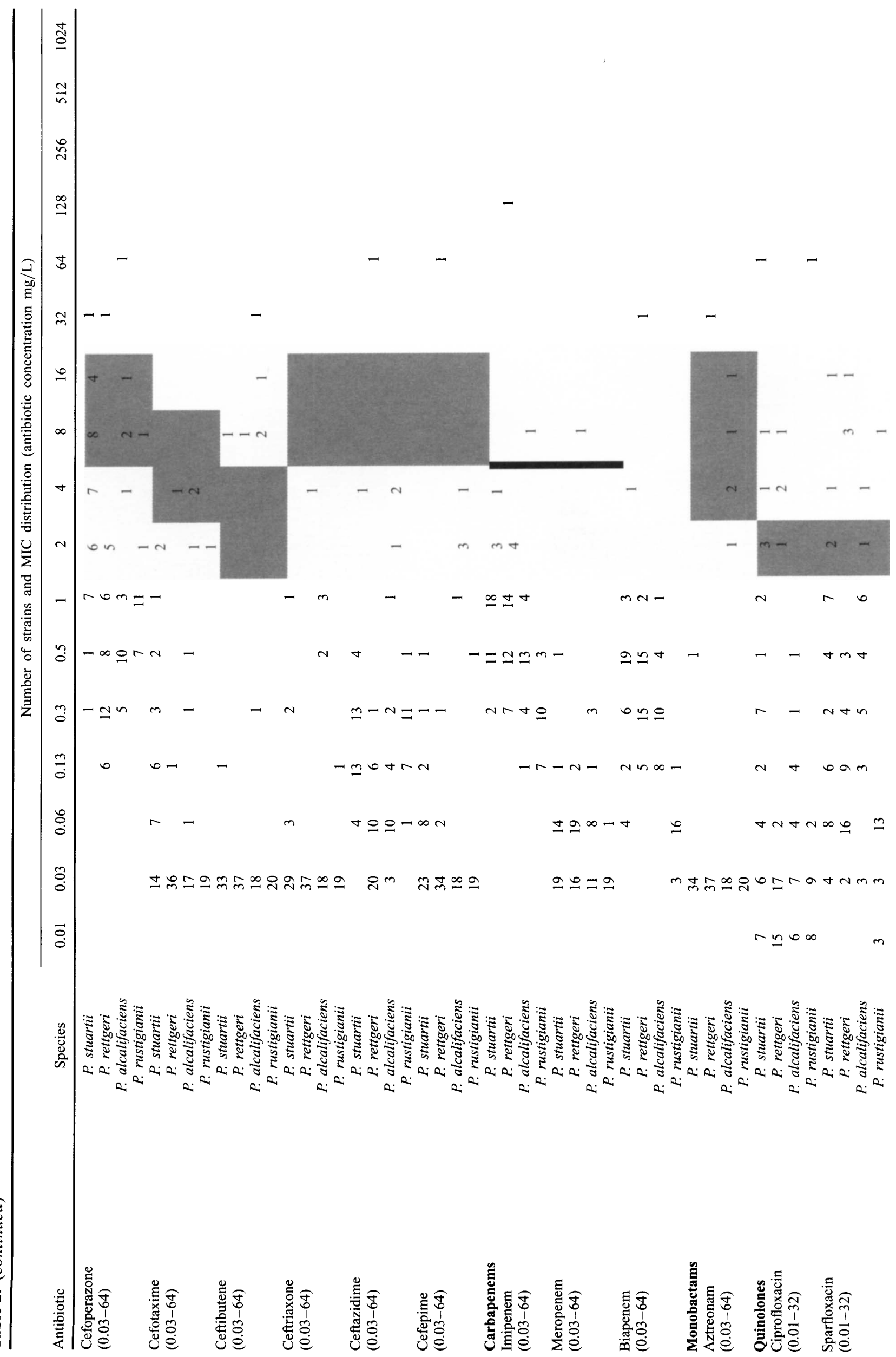




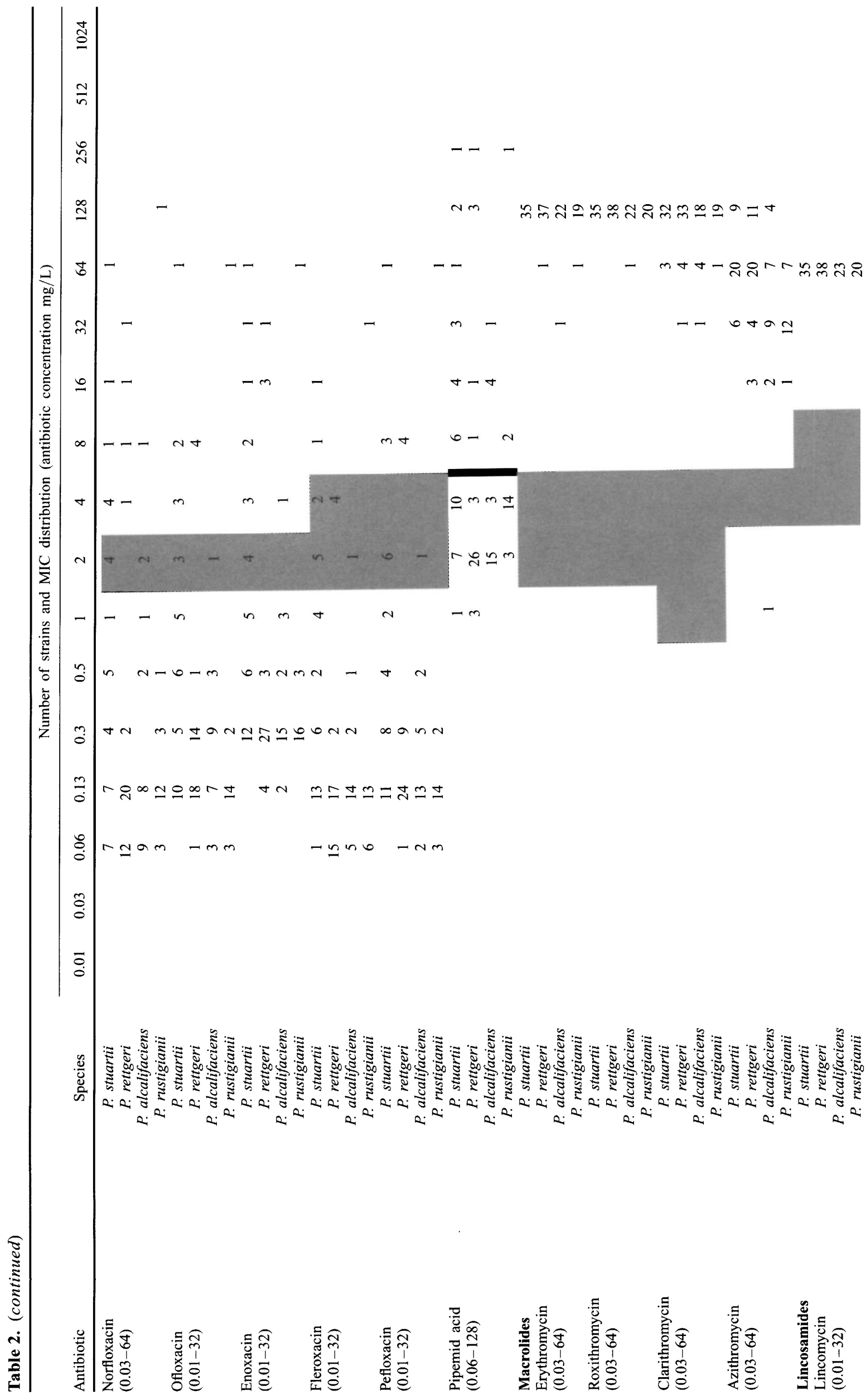




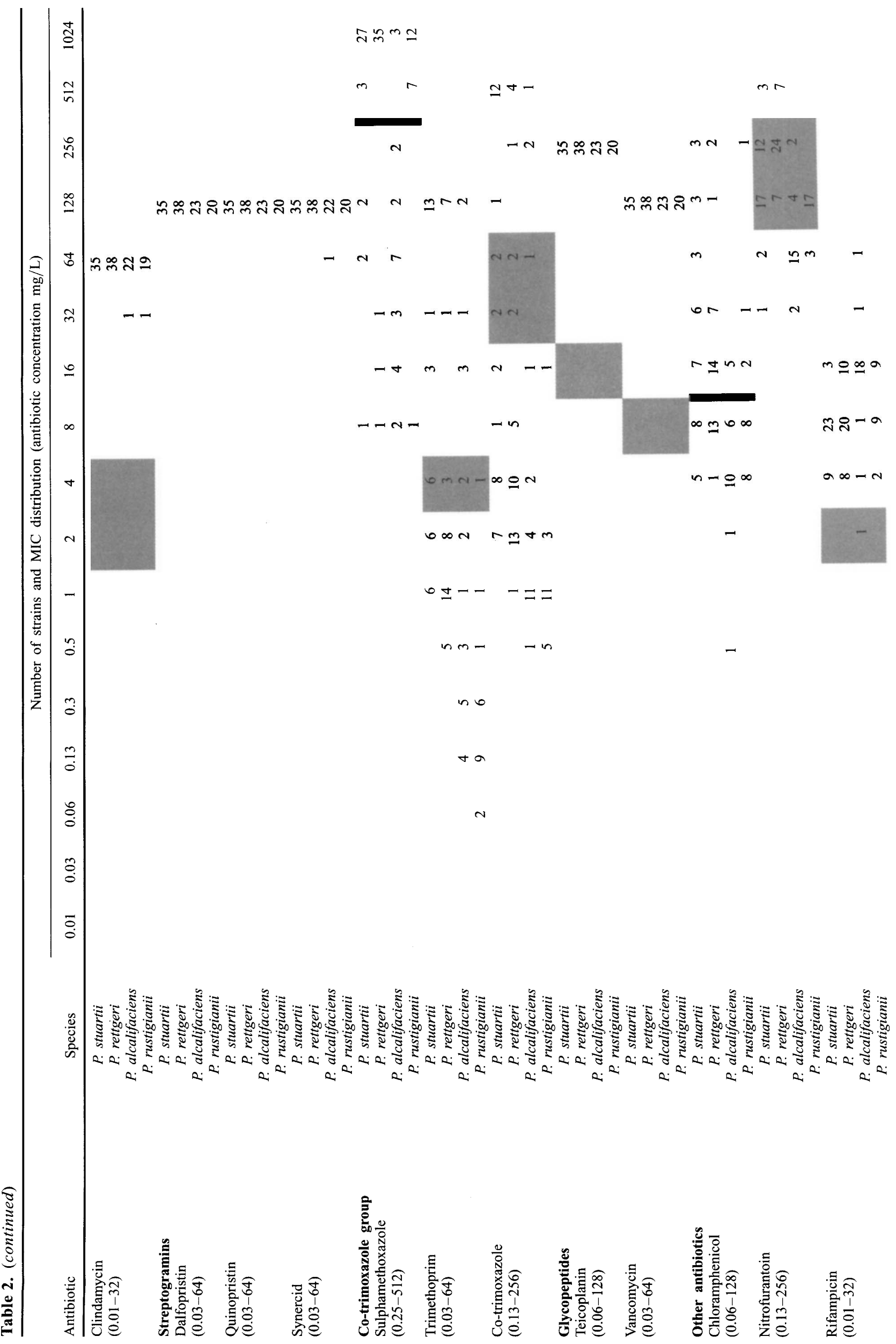




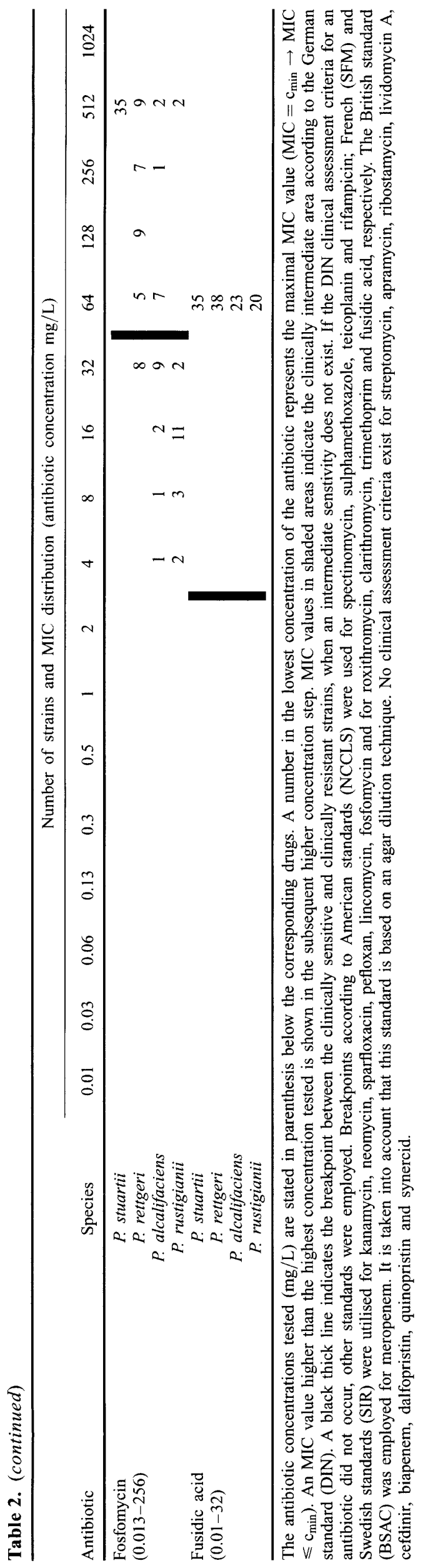

other species of Enterobacteriaceae were naturally sensitive or naturally of intermediate susceptibility. However, there was also a range of antibiotics, such as modern penicillins and cephalosporins, carbapenems and aztreonam, to which the species was naturally sensitive. The natural susceptibility pattern to some antibiotics, especially aminoglycosides and quiniolones, was difficult to assess. The MIC distributions of quinolones did not correspond to standard distributions and unequivocal clinical interpretations of the data could not be made. Although clinical interpretations of the MIC distributions of aminoglycosides seemed to be established easily, $P$ stuartii should be regarded as naturally resistant to certain aminoglycosides (see below).

According to the data, $P$ alcalifaciens and $P$. rustigianii were the most susceptible Providencia spp. They were naturally sensitive or naturally of intermediate susceptibility to tetracyclines and sensitive to aminoglycosides and quinolones. Both species were also naturally sensitive to antibiotics to which $P$. stuartii was naturally sensitive. Susceptibility to sparfloxacin, biapenem and sulphamethoxazole were suitable parameters for the discrimination of $P$. alcalifaciens and P. rustigianii (Tables 2 and 3). The natural antibiotic susceptibility patterns of $P$. rettgeri were between those of $P$. stuartii and those of the other Providencia spp. Like $P$. stuartii, $P$. rettgeri was resistant to tetracyclines and fosfomycin, but was more susceptible to aminoglycosides, quinolones, fosfomycin and numerous $\beta$-lactam antibiotics (Tables 2 and 3 ).

Most resistance mechanisms (including natural resistance) to antimicrobial agents in Providencia spp. are unknown. This is particularly true for species other than $P$. stuartii. Resistance to aminoglycosides is mostly attributed to the action of aminoglycosidemodifying enzymes. In $P$. stuartii, natural resistance to aminoglycosides is limited to aminoglycosides of the gentamicin type (gentamicin, tobramycin and netilmicin) and is due to a chromosomally encoded $2^{\prime}-\mathrm{N}$ acetyltransferase, designed $\mathrm{AAC}\left(2^{\prime}\right)$-Ia, which is distributed ubiquitously in this species [16]. According to the present data $P$. stuartii was sensitive, intermediate or slightly resistant (depending on the applied standard) to gentamicin (Table 3), but there was no uniform resistance. Otherwise, the species showed significantly less susceptibility to gentamicin, tobramycin and netilmicin than the other Providencia spp. (Table 2). It has been shown that the acetyltransferase is normally expressed at low levels, but high-level expression confers resistance to gentamicin and structurally related aminoglycosides [16]. However, the MIC data did not confirm that the AAC $\left(2^{\prime}\right)$-Ia enzyme was also present in $P$ rettgeri, as has been described $[17,18]$.

Excluding resistance to oxacillin, and presumably benzylpenicillin, natural resistance to $\beta$-lactam agents in Providencia strains is probably due to inducible 
Table 3. Grouping of the natural population of $P$. stuartii, P. rettgeri, $P$. alcalifaciens and $P$. rustigianii into the categories sensitive, intermediate and resistant

\begin{tabular}{|c|c|c|c|c|c|c|c|c|c|c|c|c|c|c|c|c|c|}
\hline \multirow{2}{*}{$\begin{array}{l}\text { Antibiotic } \\
\text { group }\end{array}$} & \multirow[b]{2}{*}{ Antibiotic } & \multirow[b]{2}{*}{ Taxon } & & $\begin{array}{l}\mathrm{Nat} \\
\text { sen }\end{array}$ & $\begin{array}{l}\text { tura } \\
\text { asiti }\end{array}$ & $\begin{array}{l}\text { lly } \\
\text { ve }\end{array}$ & & & $\begin{array}{c}\mathrm{Nat} \\
\text { nter }\end{array}$ & tura & $\begin{array}{l}\text { lly } \\
\text { diate }\end{array}$ & & & & $\begin{array}{l}\text { tura } \\
\text { sista }\end{array}$ & $\begin{array}{l}\text { ally } \\
\text { ant }\end{array}$ & \\
\hline & & & $\mathbf{F}$ & $\mathrm{S}$ & G & $\mathrm{U}$ & B & $\mathrm{F}$ & $\mathrm{S}$ & G & $\mathrm{U}$ & $\mathrm{B}$ & $\mathrm{F}$ & $\mathrm{S}$ & G & $\mathrm{U}$ & B \\
\hline Tetracyclines & Tetracycline & P. stuartii & & & & & & & & & & & $\mathrm{x}$ & $\mathrm{x}$ & $\mathrm{x}$ & $\mathrm{x}$ & $\mathrm{x}$ \\
\hline & & $P$. rettgeri & & & & & & $\mathrm{x}$ & & & $\mathrm{x}$ & & & $\mathrm{x}$ & $\mathrm{x}$ & & $\mathrm{x}$ \\
\hline & & $P$ alcalifaciens, $P$. rustigianii & $\mathrm{x}$ & & & $\mathrm{x}$ & $\mathrm{x}$ & & $\mathrm{x}$ & $\mathrm{x}$ & & & & $\mathrm{x}$ & & & $\mathrm{x}$ \\
\hline & Doxycycline & $P$. stuartii, $P$. rettgeri & & & & & & & & & & & $\mathrm{x}$ & $\mathrm{x}$ & $\mathrm{x}$ & & $\mathrm{x}$ \\
\hline & & P. alcalifaciens, $P$. rustigianii & $\mathrm{x}$ & & & & $\mathrm{x}$ & & $\mathrm{x}$ & $\mathrm{x}$ & & & & $\mathrm{x}$ & & & $\mathrm{x}$ \\
\hline & Minocycline & P. stuartii & & & & & & & & & & & $\mathrm{x}$ & & $\mathrm{x}$ & & $\mathrm{x}$ \\
\hline & & P. rettgeri & $\mathrm{x}$ & & & & & $\mathrm{x}$ & & & & & $\mathrm{x}$ & & $\mathrm{x}$ & & $\mathrm{x}$ \\
\hline & & P. alcalifaciens & $\mathrm{x}$ & & & & $\mathrm{x}$ & $\mathrm{x}$ & & $\mathrm{x}$ & & & $\mathrm{x}$ & & $\mathrm{x}$ & & $\mathrm{x}$ \\
\hline & & P. rustigianii & $\mathrm{x}$ & & & & $\mathrm{x}$ & & & $\mathrm{x}$ & & & & & & & $\mathrm{x}$ \\
\hline Aminoglycosides & Amikacin & All species & $\mathrm{x}$ & $\mathrm{x}$ & $\mathbf{x}$ & $\mathrm{x}$ & $\mathrm{x}$ & & & & & & & & & & \\
\hline & Gentamicin & P. stuartii & $\mathrm{x}$ & $\mathrm{x}$ & $\mathrm{x}$ & $\mathrm{x}$ & $\mathrm{x}$ & $\mathrm{x}$ & & $\mathrm{x}$ & $\mathrm{x}$ & $\mathrm{x}$ & & $\mathrm{x}$ & $\mathrm{x}$ & & $\mathrm{x}$ \\
\hline & & P. rettgeri & $\mathrm{x}$ & $\mathrm{x}$ & $\mathrm{x}$ & $\mathrm{x}$ & $\mathrm{x}$ & & & $\mathrm{x}$ & & $\mathrm{x}$ & & & & & \\
\hline & & $P$ alcalifaciens, $P$. rustigianii & $\mathrm{x}$ & $\mathrm{x}$ & $\mathrm{x}$ & $\mathrm{x}$ & $\mathrm{x}$ & & & & & & & & & & \\
\hline & Netilmicin & P. stuartii & $\mathrm{x}$ & $\mathrm{x}$ & & $\mathrm{x}$ & $\mathrm{x}$ & $\mathrm{x}$ & & $\mathrm{x}$ & & $\mathrm{x}$ & & $\mathrm{x}$ & $\mathrm{x}$ & & $\mathrm{x}$ \\
\hline & & $P$. rettgeri & $\mathrm{x}$ & $\mathrm{x}$ & $\mathrm{x}$ & $\mathrm{x}$ & $\mathrm{x}$ & & & $\mathrm{x}$ & & $\mathrm{x}$ & & & & & \\
\hline & & P. alcalifaciens, $P$. mustigianii & $\mathrm{x}$ & $\mathrm{x}$ & $\mathrm{x}$ & $\mathrm{x}$ & $\mathrm{x}$ & & & & & & & & & & \\
\hline & Tobramycin & P. stuartii & $\mathrm{x}$ & $\mathrm{x}$ & $\mathrm{x}$ & $\mathrm{x}$ & $\mathrm{x}$ & $\mathrm{x}$ & $\mathrm{x}$ & $\mathrm{x}$ & $\mathrm{x}$ & $\mathrm{x}$ & $\mathrm{x}$ & $\mathrm{x}$ & $\mathrm{x}$ & $\mathrm{x}$ & $\mathrm{x}$ \\
\hline & & P. rettgeri & $\mathrm{x}$ & $\mathrm{x}$ & $\mathrm{x}$ & $\mathrm{x}$ & $\mathrm{x}$ & & & $\mathrm{x}$ & & $\mathrm{x}$ & & & & & \\
\hline & & $P$. alcalifaciens, $P$. rustigianii & $\mathbf{x}$ & $\mathrm{x}$ & $\mathrm{x}$ & $\mathrm{x}$ & $\mathrm{x}$ & & & & & & & & & & \\
\hline & Kanamycin & All species & $\mathrm{x}$ & & & $\mathrm{x}$ & & & & & & & & & & & \\
\hline & Neomycin & P. stuartii & $\mathrm{x}$ & & & & & $\mathrm{x}$ & & & & & $\mathrm{x}$ & & & & \\
\hline & & P. rettgeri & $\mathrm{x}$ & & & & & $\mathrm{x}$ & & & & & & & & & \\
\hline & & $P$ alcalifaciens, $P$. rustigianii & $\mathrm{x}$ & & & & & & & & & & & & & & \\
\hline & Spectinomycin & P. stuartii & & & & & & & & & & & & & & $\mathrm{x}$ & \\
\hline & & $P$. rettgeri & & & & $\mathrm{x}$ & & & & & & & & & & $\mathrm{x}$ & \\
\hline & & $P$. alcalifaciens, $P$. rustigianii & & & & $\mathrm{x}$ & & & & & & & & & & & \\
\hline Penicillins & Benzylpenicillin & P. stuartii & & & & & & & & & & & $\mathrm{x}$ & $\mathrm{x}$ & $\mathrm{x}$ & $\mathrm{x}$ & \\
\hline & & P. rettgeri & & & & & & $\mathrm{x}$ & $\mathrm{x}$ & & $\mathrm{x}$ & & $\mathrm{x}$ & $\mathrm{x}$ & $\mathrm{x}$ & $\mathrm{x}$ & \\
\hline & & $P$ alcalifaciens & & & & & & $\mathrm{x}$ & $\mathrm{x}$ & & & & $\mathrm{x}$ & $\mathrm{x}$ & $\mathrm{x}$ & $\mathrm{x}$ & \\
\hline & & P. rustigianii & & & & & & $\mathrm{x}$ & $\mathrm{x}$ & & & & & $\mathbf{x}$ & $\mathrm{x}$ & $\mathrm{x}$ & \\
\hline & Oxacillin & All species & & & & & & & & & & & & $x$ & $\mathrm{x}$ & $\mathrm{x}$ & \\
\hline & Amoxycillin & P. stuartii & & & & & & & & & & & $\mathrm{x}$ & $\mathrm{x}$ & $\mathrm{x}$ & $\mathrm{x}$ & $\mathrm{x}$ \\
\hline & & P. rettgeri & $\mathrm{x}$ & $\mathrm{x}$ & $\mathrm{x}$ & $\mathrm{x}$ & $\mathrm{x}$ & $\mathrm{x}$ & $\mathrm{x}$ & $\mathrm{x}$ & $\mathrm{x}$ & & $\mathrm{x}$ & $\mathrm{x}$ & $\mathrm{x}$ & $\mathrm{x}$ & $\mathrm{x}$ \\
\hline & & P. alcalifaciens & & & & $\mathrm{x}$ & $\mathrm{x}$ & $\mathrm{x}$ & $\mathrm{x}$ & $\mathrm{x}$ & $\mathbf{x}$ & & $\mathrm{x}$ & $\mathrm{x}$ & $\mathrm{x}$ & $\mathrm{x}$ & $\mathrm{x}$ \\
\hline & & P. rustigianii & $\mathrm{x}$ & & $\mathrm{x}$ & $\mathrm{x}$ & $\mathbf{x}$ & $\mathrm{x}$ & $\mathrm{x}$ & $\mathrm{x}$ & $\mathrm{x}$ & & & $\mathrm{x}$ & $\mathrm{x}$ & & $\mathrm{x}$ \\
\hline & AMX/CLAV & P. stuartii & & & & & & $\mathrm{x}$ & & & $\mathrm{x}$ & & $\mathrm{x}$ & $\mathrm{x}$ & $\mathrm{x}$ & $\mathrm{x}$ & $\mathrm{x}$ \\
\hline & & All species except $P$ stuartii & $\mathrm{x}$ & & $\mathbf{x}$ & $\mathrm{x}$ & $\mathrm{x}$ & $\mathrm{x}$ & $\mathrm{x}$ & $\mathrm{x}$ & $\mathrm{x}$ & & $\mathrm{x}$ & $\mathrm{x}$ & $\mathrm{x}$ & $\mathrm{x}$ & $\mathrm{x}$ \\
\hline & AMP/SUL & P. stuartii & & & & $\mathrm{x}$ & $\mathrm{x}$ & $\mathbf{x}$ & $\mathrm{x}$ & $\mathrm{x}$ & $\mathrm{x}$ & & & $\mathrm{x}$ & $\mathrm{x}$ & & $\mathrm{x}$ \\
\hline & & P. rettgeri & $\mathrm{x}$ & $\mathrm{x}$ & $\mathrm{x}$ & $\mathbf{x}$ & $\mathrm{x}$ & $\mathrm{x}$ & $\mathrm{x}$ & $\mathrm{x}$ & $\mathrm{x}$ & & $\mathrm{x}$ & $\mathrm{x}$ & $\mathrm{x}$ & $\mathrm{x}$ & $\mathrm{x}$ \\
\hline & & P. alcalifaciens & $\mathrm{x}$ & $\mathrm{x}$ & $\mathrm{x}$ & $\mathrm{x}$ & $\mathrm{x}$ & $\mathrm{x}$ & $\mathrm{x}$ & $\mathrm{x}$ & $\mathrm{x}$ & & & $\mathrm{x}$ & $\mathrm{x}$ & & $\mathrm{x}$ \\
\hline & & P. rustigianii & $\mathrm{x}$ & $\mathrm{x}$ & $\mathrm{x}$ & $\mathrm{x}$ & $\mathrm{x}$ & & $\mathrm{x}$ & $\mathrm{x}$ & & & & & & & \\
\hline & Piperacillin & All species except $P$. alcalifaciens & $\mathrm{x}$ & $\mathrm{x}$ & $\mathrm{x}$ & $\mathrm{x}$ & $\mathrm{x}$ & & & & & & & & & & \\
\hline & & $P$ alcalifaciens & $\mathrm{x}$ & $\mathrm{x}$ & $\mathrm{x}$ & $\mathrm{x}$ & $\mathrm{x}$ & & & $\mathrm{x}$ & & & & & & & \\
\hline & PIP/TAZ & All species & $\mathrm{x}$ & $\mathbf{x}$ & $\mathrm{x}$ & $\mathrm{x}$ & $\mathrm{x}$ & & & & & & & & & & \\
\hline & Ticarcillin & All species except $P$. alcalifaciens & $\mathrm{x}$ & & $\mathrm{x}$ & $\mathrm{x}$ & $\mathrm{x}$ & & & & & & & & & & \\
\hline & & $P$. alcalifaciens & $\mathrm{x}$ & & $\mathrm{x}$ & $\mathrm{x}$ & $\mathrm{x}$ & & & $\mathrm{x}$ & & & & & & & \\
\hline & Mezlocillin & All species except $P$ alcalifaciens & $\mathrm{x}$ & $\mathrm{x}$ & $\mathrm{x}$ & $\mathrm{x}$ & $\mathrm{x}$ & & & & & & & & & & \\
\hline & & $P$ alcalifaciens & $\mathrm{x}$ & $\mathrm{x}$ & $\mathrm{x}$ & $\mathrm{x}$ & $\mathrm{x}$ & & & $\mathrm{x}$ & & & & & & & \\
\hline & Azlocillin & P. stuartii & & & $\mathrm{x}$ & $\mathrm{x}$ & $\mathrm{x}$ & & & $\mathrm{x}$ & & $\mathrm{x}$ & & & $\mathrm{x}$ & $\mathrm{x}$ & $\mathrm{x}$ \\
\hline & & $P$ rettgeri, $P$. rustigianii & & & $\mathrm{x}$ & $\mathrm{x}$ & $\mathrm{x}$ & & & & & & & & & & \\
\hline & & $P$. alcalifaciens & & & $\mathrm{x}$ & $\mathrm{x}$ & $\mathrm{x}$ & & & $\mathbf{x}$ & & $\mathrm{x}$ & & & & & \\
\hline Cephalosporins & Cefaclor & P. stuartii & & & & & & & & & $\mathrm{x}$ & & $\mathrm{x}$ & $\mathrm{x}$ & $\mathrm{x}$ & $\mathrm{x}$ & \\
\hline & & $P$. rettgeri, $P$. alcalifaciens & $\mathrm{x}$ & $\mathrm{x}$ & $\mathrm{x}$ & $\mathrm{x}$ & & $\mathrm{x}$ & $\mathrm{x}$ & $\mathrm{x}$ & $\mathrm{x}$ & & $\mathrm{x}$ & $\mathrm{x}$ & $\mathrm{x}$ & $\mathrm{x}$ & \\
\hline & & P. rustigianii & $\mathrm{x}$ & $\mathrm{x}$ & $\mathrm{x}$ & $\mathrm{x}$ & & $\mathrm{x}$ & $\mathrm{x}$ & $\mathrm{x}$ & & & & & $\mathrm{x}$ & & \\
\hline & Cefazoline & P. stuartii & & & & & & & & & & & & & $\mathrm{x}$ & $\mathrm{x}$ & \\
\hline & & $P$ rettgeri, $P$ alcalifaciens & & & $\mathrm{x}$ & $\mathrm{x}$ & & & & $\mathrm{x}$ & $\mathrm{x}$ & & & & $\mathrm{x}$ & $\mathrm{x}$ & \\
\hline & & $P$ rustigianii & & & $\mathrm{x}$ & $\mathrm{x}$ & & & & & & & & & & & \\
\hline & Loracarbef & P. stuartii & & & & $\mathrm{x}$ & & $\mathrm{x}$ & & $\mathrm{x}$ & $\mathrm{x}$ & & $\mathrm{x}$ & & $\mathrm{x}$ & $\mathrm{x}$ & \\
\hline & & P. rettgeri, $P$. alcalifaciens & $\mathrm{x}$ & & $\mathrm{x}$ & $\mathrm{x}$ & & $\mathrm{x}$ & & $\mathrm{x}$ & $\mathrm{x}$ & & $\mathrm{x}$ & & $\mathrm{x}$ & $\mathrm{x}$ & \\
\hline & & P. rustigianii & $\mathrm{x}$ & & $\mathrm{x}$ & $\mathrm{x}$ & & & & & & & & & & & \\
\hline & Cefuroxime & All species except $P$ stuartii & $\mathrm{x}$ & $\mathrm{x}$ & $\mathrm{x}$ & $\mathrm{x}$ & $\mathrm{x}$ & & & & & & & & & & \\
\hline & & P. stuartii & $\mathbf{x}$ & $\mathrm{x}$ & $\mathrm{x}$ & $\mathrm{x}$ & $\mathrm{x}$ & & & $\mathrm{x}$ & & $\mathrm{x}$ & & & & & \\
\hline & Cefotiam & All species & $\mathrm{x}$ & & $\mathrm{x}$ & $\mathbf{x}$ & & & & & & & & & & & \\
\hline & Cefetamet & All species & $\mathrm{x}$ & $\mathrm{x}$ & & & $\mathrm{x}$ & & & & & & & & & & \\
\hline & Cefoxitin & P. rustigianii & $\mathrm{x}$ & $\mathrm{x}$ & $\mathrm{x}$ & $\mathrm{x}$ & & & & & & & & & & & \\
\hline & & All species except $P$. rustigianii & $\mathrm{x}$ & $\mathrm{x}$ & $\mathrm{x}$ & $\mathrm{x}$ & & & & $\mathrm{x}$ & & & & & & & \\
\hline & Cefixim & All species & $\mathrm{x}$ & & $\mathrm{x}$ & $\mathrm{x}$ & $\mathrm{x}$ & & & & & & & & & & \\
\hline & Cefpodoxime & All species & $\mathrm{x}$ & $\mathrm{x}$ & $\mathrm{x}$ & $\mathrm{x}$ & $\mathrm{x}$ & & & & & & & & & & \\
\hline & Cefoperazone & All species except $P$. stuartii & $\mathrm{x}$ & & $\mathrm{x}$ & $\mathrm{x}$ & & & & & & & & & & & \\
\hline & & P. stuartii & $\mathbf{x}$ & & $\mathrm{x}$ & $\mathrm{x}$ & & & & $\mathrm{x}$ & & & & & & & \\
\hline & Cefotaxime & All species & $\mathrm{x}$ & $\mathrm{x}$ & $\mathrm{x}$ & $\mathrm{x}$ & $\mathrm{x}$ & & & & & & & & & & \\
\hline & Ceftibutene & All species & $\mathrm{x}$ & & $\mathrm{x}$ & $\mathrm{x}$ & & & & & & & & & & & \\
\hline
\end{tabular}


Table 3. (continued)

\begin{tabular}{|c|c|c|c|c|c|c|c|c|c|c|c|c|c|c|c|c|c|}
\hline \multirow{2}{*}{$\begin{array}{l}\text { Antibiotic } \\
\text { group }\end{array}$} & \multirow[b]{2}{*}{ Antibiotic } & \multirow[b]{2}{*}{ Taxon } & \multicolumn{5}{|c|}{$\begin{array}{l}\text { Naturally } \\
\text { sensitive }\end{array}$} & \multicolumn{5}{|c|}{$\begin{array}{c}\text { Naturally } \\
\text { intermediate }\end{array}$} & \multicolumn{5}{|c|}{$\begin{array}{l}\text { Naturally } \\
\text { resistant }\end{array}$} \\
\hline & & & $\mathrm{F}$ & $\mathrm{S}$ & G & $\mathrm{U}$ & $\mathrm{B}$ & $\mathrm{F}$ & $\mathrm{S}$ & $\mathrm{G}$ & $\mathrm{U}$ & $\mathrm{B}$ & $\mathrm{F}$ & $\mathrm{S}$ & G & $\mathrm{U}$ & B \\
\hline \multirow{5}{*}{ Carbapenems } & Ceftriaxone & All species & $\mathrm{x}$ & & $\mathrm{x}$ & $\mathrm{x}$ & & & & & & & & & & & \\
\hline & Ceftazidime & All species & $\mathrm{x}$ & $\mathrm{x}$ & $\mathrm{x}$ & $\mathrm{x}$ & $\mathrm{x}$ & & & & & & & & & & \\
\hline & Cefepime & All species & $\mathrm{x}$ & & $\mathrm{x}$ & $\mathrm{x}$ & & & & & & & & & & & \\
\hline & Imipenem & All species & $\mathrm{x}$ & $\mathrm{x}$ & $\mathrm{x}$ & $\mathrm{x}$ & $\mathrm{x}$ & & & & & & & & & & \\
\hline & Meropenem & All species & $\mathrm{x}$ & & & & $\mathrm{x}$ & & & & & & & & & & \\
\hline Monobactams & Aztreonam & All species & $\mathrm{x}$ & $\mathrm{x}$ & $\mathrm{x}$ & $\mathrm{x}$ & $\mathrm{x}$ & & & & & & & & & & \\
\hline \multirow[t]{16}{*}{ Quinolones } & Ciprofloxacin & All species except $P$ stuartii & $\mathrm{x}$ & $\mathrm{x}$ & $\mathrm{x}$ & $\mathrm{x}$ & $\mathrm{x}$ & & & & & & & & & & \\
\hline & & $P$. stuartii & $\mathrm{x}$ & $\mathrm{x}$ & $\mathrm{x}$ & $\mathrm{x}$ & $\mathrm{x}$ & $\mathrm{x}$ & $\mathrm{x}$ & $\mathrm{x}$ & $\mathrm{x}$ & $\mathrm{x}$ & & & & & \\
\hline & Sparfloxacin & P. stuartii, $P$. alcalifaciens & $\mathrm{x}$ & & & & & $\mathrm{x}$ & & & & & & & & & \\
\hline & & P. rettgeri, $P$. rustigianii & $\mathrm{x}$ & & & & & & & & & & & & & & \\
\hline & Norfloxacin & All species except $P$. stuartii & $\mathrm{x}$ & $\mathrm{x}$ & $\mathrm{x}$ & $\mathrm{x}$ & $\mathrm{x}$ & & & & & & & & & & \\
\hline & & P. stuartii & $\mathrm{x}$ & $\mathrm{x}$ & $\mathrm{x}$ & $\mathrm{x}$ & $\mathrm{x}$ & $\mathrm{x}$ & $\mathrm{x}$ & $\mathrm{x}$ & & $\mathrm{x}$ & & & $\mathrm{x}$ & & $\mathrm{x}$ \\
\hline & Ofloxacin & All species except $P$. stuartii & $\mathrm{x}$ & $\mathrm{x}$ & $\mathrm{x}$ & $\mathrm{x}$ & $\mathrm{x}$ & & & & & & & & & & \\
\hline & & P. stuartii & $\mathrm{x}$ & $\mathrm{x}$ & $\mathrm{x}$ & $\mathrm{x}$ & $\mathrm{x}$ & $\mathrm{x}$ & $\mathrm{x}$ & $\mathrm{x}$ & $\mathrm{x}$ & $\mathrm{x}$ & & & $\mathrm{x}$ & & \\
\hline & Enoxacin & All species except $P$. stuartii & $\mathrm{x}$ & & $\mathrm{x}$ & $\mathrm{x}$ & $\mathrm{x}$ & & & & & & & & & & \\
\hline & & $P$ stuartii & $\mathrm{x}$ & & $\mathrm{x}$ & $\mathrm{x}$ & $\mathrm{x}$ & $\mathrm{x}$ & & $\mathrm{x}$ & $\mathrm{x}$ & $\mathrm{x}$ & $\mathrm{x}$ & & $\mathrm{x}$ & $\mathrm{x}$ & $\mathrm{x}$ \\
\hline & Fleroxacin & All species except $P$ stuartii & & & $\mathrm{x}$ & $\mathrm{x}$ & & & & & & & & & & & \\
\hline & & $P$ stuartii & & & $\mathrm{x}$ & $\mathrm{x}$ & & & & $\mathrm{x}$ & $\mathrm{x}$ & & & & & & \\
\hline & Pefloxacin & All species except $P$ stuartii & $\mathrm{x}$ & & & & & & & & & & & & & & \\
\hline & & $P$ stuartii & $\mathrm{x}$ & & & & & $\mathrm{x}$ & & & & & & & & & \\
\hline & Pipemid acid & All species except $P$ stuartii & $\mathrm{x}$ & & $\mathrm{x}$ & & & & & & & & $\mathrm{x}$ & & $\mathrm{x}$ & & \\
\hline & & P. stuartii & $\mathrm{x}$ & & $\mathbf{x}$ & & & $\mathrm{x}$ & & & & & & & $\mathrm{x}$ & & \\
\hline \multirow[t]{4}{*}{ Macrolides } & Erythromycin & All species & & & & & & & & & & & $\mathrm{x}$ & $\mathrm{x}$ & $\mathrm{x}$ & $\mathrm{x}$ & \\
\hline & Roxithromycin & All species & & & & & & & & & & & $\mathrm{x}$ & $\mathrm{x}$ & & & \\
\hline & Clarithromycin & All species & & & & & & & & & & & $\mathrm{x}$ & $\mathrm{x}$ & & $\mathrm{x}$ & \\
\hline & Azithromycin & All species & & & & & & & & & & & $\mathbf{x}$ & & $\mathrm{x}$ & $\mathrm{x}$ & \\
\hline \multirow[t]{2}{*}{ Lincosamides } & Lincomycin & All species & & & & & & & & & & & $\mathrm{x}$ & & & & \\
\hline & Clindamycin & All species & & & & & & & & & & & $\mathrm{x}$ & $\mathrm{x}$ & $\mathrm{x}$ & $\mathrm{x}$ & \\
\hline \multirow[t]{6}{*}{ Co-trimoxazole group } & Sulphamethoxazole & All species except $P$. alcalifaciens & & & & & & & & & & & & & & $\mathrm{x}$ & $\mathrm{x}$ \\
\hline & & $P$ alcalifaciens & & & & $\mathrm{x}$ & $\mathrm{x}$ & & & & & & & & & & $\mathrm{x}$ \\
\hline & Trimethoprim & P. stuartii & $\mathrm{x}$ & $\mathrm{x}$ & & $\mathrm{x}$ & & & $\mathrm{x}$ & & & $\mathrm{x}$ & & & & & $\mathrm{x}$ \\
\hline & & $P$ rettgeri, $P$. alcalifaciens & $\mathrm{x}$ & $\mathrm{x}$ & & $\mathrm{x}$ & $\mathrm{x}$ & & $\mathrm{x}$ & & & $\mathrm{x}$ & & & & & $\mathrm{x}$ \\
\hline & & P. rustigianii & $\mathrm{x}$ & $\mathrm{x}$ & & $\mathrm{x}$ & $\mathrm{x}$ & & & & & & & & & & \\
\hline & Co-trimoxazole & All species & $\mathrm{x}$ & $\mathrm{x}$ & $\mathrm{x}$ & $\mathrm{x}$ & & & & & & & & & & & \\
\hline \multirow[t]{2}{*}{ Glycopeptides } & Teicoplanin & All species & & & & & & & & & & & $\mathrm{x}$ & & & $\mathrm{x}$ & \\
\hline & Vancomycin & All species & & & & & & & & & & & $\mathbf{x}$ & $\mathrm{x}$ & $\mathrm{x}$ & $\mathrm{x}$ & \\
\hline \multirow{13}{*}{ Other antibiotics } & Chloramphenicol & P. stuartii, $P$. rettgeri & $\mathrm{x}$ & $\mathrm{x}$ & $\mathrm{x}$ & $\mathrm{x}$ & $\mathrm{x}$ & $\mathrm{x}$ & & & $\mathrm{x}$ & & $\mathrm{x}$ & $\mathrm{x}$ & $\mathrm{x}$ & $\mathrm{x}$ & $\mathrm{x}$ \\
\hline & & $P$. alcalifaciens, $P$. rustigianii & $\mathrm{x}$ & $\mathrm{x}$ & $\mathrm{x}$ & $\mathrm{x}$ & $\mathrm{x}$ & $\mathrm{x}$ & & & $\mathrm{x}$ & & & $\mathrm{x}$ & $\mathrm{x}$ & & $\mathrm{x}$ \\
\hline & Nitrofurantoin & P. stuartii & & & $\mathrm{x}$ & & & $\mathrm{x}$ & & $\mathrm{x}$ & $\mathrm{x}$ & & $\mathrm{x}$ & $\mathrm{x}$ & $\mathrm{x}$ & $\mathrm{x}$ & \\
\hline & & P. rettgeri & & & & & & $\mathrm{x}$ & & $\mathrm{x}$ & & & $\mathrm{x}$ & $\mathrm{x}$ & $\mathrm{x}$ & $\mathrm{x}$ & \\
\hline & & $P$ alcalifaciens & & $\mathrm{x}$ & $\mathrm{x}$ & $\mathrm{x}$ & & $\mathrm{x}$ & & $\mathrm{x}$ & $\mathrm{x}$ & & $\mathrm{x}$ & $\mathrm{x}$ & $\mathrm{x}$ & $\mathrm{x}$ & \\
\hline & & $P$ rustigianii & & & $\mathrm{x}$ & & & $\mathrm{x}$ & & & $\mathrm{x}$ & & & $\mathrm{x}$ & $\mathrm{x}$ & $\mathrm{x}$ & \\
\hline & Rifampicin & All species except $P$. alcalifaciens & $\mathrm{x}$ & & & & & $\mathrm{x}$ & & & & & & $\mathrm{x}$ & & $\mathrm{x}$ & \\
\hline & & P. alcalifaciens & $\mathrm{x}$ & & & & & $\mathrm{x}$ & & & & & $\mathrm{x}$ & $\mathrm{x}$ & & $\mathrm{x}$ & \\
\hline & Fosfomycin & P. stuartii & & & & & & & & & & & $\mathrm{x}$ & $\mathrm{x}$ & & & \\
\hline & & P. rettgeri & $\mathrm{x}$ & $\mathrm{x}$ & & & & & & & & & $\mathrm{x}$ & $\mathrm{x}$ & & & \\
\hline & & $P$ alcalifaciens & $\mathrm{x}$ & $\mathrm{x}$ & & & & & & & & & $\mathrm{x}$ & & & & \\
\hline & & $P$. rustigianii & $\mathrm{x}$ & $\mathrm{x}$ & & & & & & & & & & & & & \\
\hline & Fusidic acid & All species & & & & & & & & & & & & $\mathrm{x}$ & & & \\
\hline
\end{tabular}

Grouping was done according to French (SFM, abbreviated as F), Swedish, (SIR, S), German (DIN, G), American (NCCLS, U) and British standards (BSAC, B). If no $x$ is shown, that country has not given standards for that antibiotic. It should be noted that British clinical assessment criteria are based on an agar dilution technique. Streptomycin, apramycin, ribostamycin, lividomycin A, cefdinir, biapenem, dalfopristin, quinopristin and synercid are not mentioned in this table, because clinical assessment criteria do not exist for these antibiotics. AMX/CLAV, amoxycillin/clavulanate; AMP/SUL, ampicillin/sulbactam; PIP/TAZ, piperacillin/tazobactam.

chromosomal $\beta$-lactamases, which are not yet characterised. Among providenciae the natural susceptibility to a wide range of $\beta$-lactam agents was difficult to determine, because the MIC ranges were often very broad (as was found with natural susceptibility to quinolones among $P$. stuartii strains). This was particularly true for aminopenicillins and some first and second generation cephalosporins (cefaclor, cefazolin and loracarbef), and also for ticarcillin against $P$. alcalifaciens (Table 2). Small variations in the inoculum size caused alterations of the MIC values over several concentration intervals. With an inoculum size of $10^{6} \mathrm{cfu} / \mathrm{ml}$, several strains of all species were also resistant to third and fourth generation cephalosporins, such as ceftazidime and cefepime (data not shown). However, results from repeated MIC determinations with a constant inoculum were also variable. This might be due to variable inducibility of $\beta$ lactamase expression in a Providencia strain. The molecular basis of this phenomenon remains to be elucidated.

The data represent an assessment of the natural susceptibility of strains of Providencia spp. to a wide 
range of antibiotics. These databases can be used for the validation of antibiotic susceptibility test results of Providencia spp., especially with those antibiotics towards which the species are naturally resistant. If Providencia strains are found that are sensitive to these antibiotics, the antibiotic susceptibility testing should be repeated and the identification results confirmed.

We gratefully acknowledge generous support from MERLINDiagnostika, Bornheim, Germany.

\section{References}

1. Hawkey PM. Providencia stuartii: a review of a multiply antibiotic-resistant bacterium. J Antimicrob Chemother 1984; 13: $209-226$.

2. Rinker AG, Boyd AL, Gary ND, Kundig W. Isolation of multiple antibiotic resistant Enterobacteriaceae from river water. Microbios 1988; 56: 169-175.

3. Ladds PW, Bradley J, Hirst RG. Providencia rettgeri meningitis in hatchling saltwater crocodiles (Crocodylus porosus). Aust Vet $J$ 1996; 74: 397-398.

4. Albert MJ, Alam K, Ansaruzzaman M et al. Pathogenesis of Providencia alcalifaciens-induced diarrhea. Infect Immun 1992; 60: $5017-5024$.

5. Guth BEC, Perrella E. Prevalence of invasive ability and other virulence-associated characteristics in Providencia alcalifaciens strains isolated in São Paulo, Brazil, J Med Microbiol 1996; 45: $459-462$.

6. Haynes J, Hawkey PM. Providencia alcalifaciens and travellers' diarrhoea. $B M J$ 1989; 299: 94-95.

7. Picard B, Picard-Pasquier N, Krishnamoorthy R, Goullet P. Correlation between DNA polymorphism and enzyme polymorphism argues in favour of the delineation of two species within Providencia alcalifaciens. Res Microbiol 1991; 142: 965-969.

8. Hickman-Brenner FW, Farmer JJ, Steigerwalt AG, Brenner DJ.
Providencia rustigianii: a new species in the family Enterobacteriaceae formerly known as Providencia alcalifaciens biogroup 3. J Clin Microbiol 1983; 17: 1057-1060.

9. Costas M, Holmes B, Sloss LL. Numerical analysis of electrophoretic protein patterns of Providencia rustigianii strains from human diarrhoea and other sources. $J \mathrm{Appl}$ Bacteriol 1987; 63: 319-328.

10. Müller HE, O'Hara CM, Fanning GR, Hickman-Brenner FW, Swenson JM, Brenner DJ. Providencia heimbachae, a new species of Enterobacteriaceae isolated from animals. Int $J$ Syst Bacteriol 1986; 36: 252-256.

11. Penner JL. The genera Proteus, Providencia, and Morganella. In: Balows A, Trüper GG, Dworkin M, Harder W, Schleifer KH (eds) The Prokaryotes: a handbook on the biology of bacteria: ecophysiology, isolation, identification, applications. New York, Springer-Verlag. 1992: 2849-2862.

12. Cornaglia G, Frugoni S, Mazzariol A, Piacentini E, Berlusconi A, Fontana R. Activities of oral antibiotics on Providencia strains isolated from institutionalized elderly patients with urinary tract infections. Antimicrob Agents Chemother 1995; 39: $2819-2821$.

13. Lindsey JO, Martin WT, Sonnenwirth AC, Bennet JV. An outbreak of nosocomial Proteus rettgeri urinary tract infection. Am J Epidemiol 1976; 103: 2461-2469.

14. Toni M, Casewell MW, Schito GC. Reappraisal of the significance of multiply resistant urinary isolates of Proteus rettgeri. J Antimicrob Chemother 1980; 6: 527-534.

15. Farmer JJ. Enterobacteriaceae: introduction and identification. In: Murray P, Baron E, Pfaller M, Tenover F, Yolken R (eds) Manual of clinical microbiology. Washington, DC, American Society for Microbiology. 1995: 438-449.

16. Rather PN, Orosz E, Shaw KJ, Hare R, Miller G. Characterization and transcriptional regulation of the $2^{\prime}-\mathrm{N}$-acetyltransferase gene from Providencia stuartii. J Bacteriol 1993; 175: 6492-6498.

17. Gu JW, Neu HC. In vitro activity of Dactimicin, a novel pseudodisaccharide aminoglycoside, compared with activities of other aminoglycosides. Antimicrob Agents Chemother 1989; 33: 1998-2003.

18. Hawkey PM, Penner JL, Linton AH, Hawkey CA, Crisp JL, Hinton M. Speciation, serotyping, antimicrobial sensitivity and plasmid content of Proteeae from the environment of calfrearing units in South West England. J Hyg 1986; 97: 405417. 